\title{
Dinámica espacial y temporal de la interacción de Ascomorphella volvocicola y Volvox en el embalse Riogrande II, Antioquia, primer reporte para Colombia Spatial and temporal dynamics of the interaction of Ascomorphella volvocicola and Volvox in Riogrande II reservoir in Antioquia, first report for Colombia
}

\author{
(1) Ana Lucía Estrada-Posada, 다래 Yohana Agudelo-Acevedo \\ Grupo de Limnología Básica y Experimental - LimnoBasE, Instituto de Biología, \\ Universidad de Antioquia, Medellín, Colombia
}

\begin{abstract}
Resumen
Se estudió la variación espacial y temporal de las densidades de Ascomorphella volvocicola y Volvox en el embalse Riogrande II mediante la recolección de muestras cada diez días durante 12 meses en tres sitios ubicados en el eje longitudinal del río Chico. Asimismo, se estimó el porcentaje de daño de las membranas ocasionado por el rotífero y el porcentaje de infección en las colonias del alga. La comprobación de la hipótesis de investigación indicó que el rotífero (A. volvocicola) es un endoparásito obligado que desarrolla su ciclo de vida dentro del alga hospedera (Volvox), cuya dinámica poblacional está determinada por cambios espaciales y temporales del huésped y en ningún momento por las condiciones ambientales del embalse. Por otra parte, ni el parásito ni los nutrientes ni otras variables ambientales evaluadas determinaron las variaciones de las densidades del alga colonial, probablemente debido a la capacidad de migración de Volvox en la columna de agua, lo que le permite escoger los ambientes más convenientes. Se concluyó que solo Volvox determina la aparición y la dinámica de A. volvocicola, pero este no influye en la dinámica de Volvox, ya que el rotífero endoparásito no logró diezmar las poblaciones de Volvox en el ciclo hidrológico evaluado. Se comprobó que existe una relación de dependencia en un solo sentido, de tal manera que $A$. volvocicola solo está presente si existen colonias de Volvox.
\end{abstract}

Palabras clave: rotifera; parasitismo; embalse; migración vertical; Volvocales.

\begin{abstract}
We studied the spatial and temporal variations of Ascomorphella volvocicola and Volvox densities in Riogrande II reservoir by collecting samples every 10 days for 12 months at three sites located on the longitudinal axis of the Chico River. We also estimated the percentage of membrane damage caused by the rotifer and the percentage of infection in the algae colonies. The verification of the research hypothesis indicated that the rotifer $(A$. volvocicola $)$ is an obligate endoparasite that develops its life cycle within the host algae (Volvox), whose population dynamics is determined by spatial and temporal changes of the host and at no time by the environmental conditions of the reservoir. In contrast, the variations in the density of the colonial algae were not determined by the parasite or by the nutrients or other environmental variables evaluated possibly due to the migration capacity of Volvox in the water column, which allows it to choose the most convenient environments. We concluded that only Volvox determines the appearance and dynamics of $A$. volvocicola, but this does not influence the dynamics of Volvox, as this endoparasite rotifer failed to decimate Volvox populations in the hydrological cycle evaluated. We also concluded that there is a one-way dependency relationship such that $A$. volvocicola will only be present if there are Volvox colonies.
\end{abstract}

Keywords: Rotifera; parasitism; reservoir; vertical migration; Volvocales.

*Correspondencia

Ana Lucía Estrada Posada; limnobio.2007@gmail.com; Recibido: 6 de marzo de 2019; Aceptado: 8 de agosto de 2019;Editor: Sandra Baena 


\section{Introducción}

Durante el análisis de muestras de zooplancton recolectadas en el embalse Riogrande II, Estrada-Posada (2007) encontró individuos y huevos de Ascomorphella volvocicola dentro de las colonias de Volvox sp. A partir de este hallazgo, se retomaron dichas muestras para analizar la dinámica espacial y temporal de la interacción del rotífero y la clorofita. Este tipo de relación no ha sido reportada en los embalses de Colombia y, muy probablemente, tampoco en Suramérica, pues no se encontraron publicaciones en este sentido.

En cuanto a los embalses colombianos, solo se han registrado datos de abundancia o biomasa del rotífero A. volvocicola en Riogrande II (Estrada-Posada, 2007; Villabona-González, et al., 2014) y en La Fe (VillabonaGonzález, et al., 2014). La distribución restringida de este rotífero puede estar asociada a la distribución de Volvox en los embalses colombianos, la cual, al parecer, también es limitada, pues solo se ha reportado su presencia en El Peñol (Ramírez, 1986), en La Fe (Estrada-Posada, 1999), en Riogrande II (Estrada-Posada, 2007), en Betania (MartínezSilva, 2015) y en Quimbo (Martínez, et al., 2018).

El presente estudio constituye, entonces, el primer reporte de la presencia e interacción de $A$. volvocicola y sus huevos en las colonias de Volvox sp. en los embalses colombianos.

Volvox es un alga verde multicelular y esférica que presenta diferenciación entre las células somáticas biflageladas y unas pocas células hijas, llamadas gonidias, las cuales empiezan a desarrollarse dentro de la colonia madre. Las hijas aparecen en número de 2 a 10 y solo quedan libres al romperse la colonia madre (Margalef, 1983; Kirk, 2004). Las colonias de Volvox miden entre 300 y $500 \mu \mathrm{m}$ de diámetro y contienen desde 500 hasta 50.000 células somáticas en una matriz gelatinosa que forma una esfera completa (Margalef, 1983; Nozaki, 2003).

El rotífero Ascomorphella volvocicola tiene un cuerpo en forma de barril, con una longitud total entre 120 y 160 $\mu \mathrm{m}$ y un ancho de $75 \mu \mathrm{m}$ (Koste, 1978). Autores como Edmondson (1959), Pennak (1989), Shiel \& Koste (1992), y Wallace \& Snell (2001) describen a A. volvocicola como un endoparásito obligado y exclusivo de las especies del género Volvox. En ocasiones, estas asociaciones solo se mencionan de forma general en el campo del parasitismo, lo que indica que hay algunos rotíferos que consumen ciertas especies de Volvox (Canter \& Lund, 1995).

El parasitismo es una relación obligatoria en la cual un huésped o parásito se aloja temporal o permanentemente dentro o sobre un individuo de especie diferente (huésped), del cual depende metabólicamente y le sirve de alimento (Ricklefs, 1998; Stiling, 1996; Sánchez, 2000). La mayoría de los parásitos se especializan más en sus huéspedes que los mismos depredadores que se comen la presa completa (Lampert \& Sommer, 1997; Ebert, 2005). En los ecosistemas acuáticos los parásitos pueden infestar a diferentes grupos de organismos, entre ellos, bacterias, algas, cianobacterias y peces (Lampert \& Sommer, 1997). Incluso el plancton incluye especies de vida parasitaria, algunas de las cuales se pueden encontrar en protozoarios, cladóceros, copépodos, rotíferos y larvas de dípteros como Chaoboridae (Ebert, 2005; Esteves, 2011).

Algunas especies de rotíferos exhiben asociaciones de tipo simbiótico, comensal, epizoico, epífitico o parasítico con protozoarios, poríferos, anélidos, briozoarios, equinodermos, moluscos (especialmente en las conchas y las masas de los huevos de gastrópodos acuáticos) y crustáceos (May, 1989).

Los primeros reportes de parasitismo en el plancton se registraron en la clorofícea Volvox y el rotífero Notommata parasita. En 1835 Ehrenberg escribió una nota de su singular hábito parásito en Volvox globator (May, 1989). Desde el año 1800 este rotífero se ha identificado como N. parasita (Ehrenberg, 1838), Proales parasita (Ehrenberg, 1838), y Cephalodella catellina volvocicola (Zavadovsky, 1916); actualmente, se encuentra reportado en la literatura como Ascomorphella volvocicola (Plate, 1886; May, 1989).

Los estudios sobre A.volvocicola son pocos y se han enfocado en registros de abundancia y biomasa (Villabona, et al., 2014), en tanto que en Volvox se han hecho estudios con énfasis en la genética y los aspectos molecularas, mecánicos, hidrodinámicos, morfológicos, reproductivos, $\mathrm{y}$ de distribución vertical o de migración, entre otros (Sommer \& Gliwicz, 1986; Kirk, 2005; Drescher, et al., 2009; Brumley, et al., 2015). No obstante, pocas investigaciones han evaluado la dinámica espacial y temporal de la interacción de A. volvocicola y Volvox. Wetzel (2001) consideraba que el parasitismo incrementa con frecuencia en cuerpos de agua eutróficos, sin embargo, no se cuenta con información que permita aproximarse a una valoración cuantitativa de la destrucción de las colonias de algas y la posibilidad de colapso de las poblaciones de Volvox, como lo postularon Ganf, et al. (1983), Van Donk \& Voogd (1998), y Fontaneto \& De Smet (2015).

El rotífero $A$. volvocicola es considerado un endoparásito obligado, pues vive dentro de las colonias de Volvox alimentándose de las células somáticas (membrana envolvente de la colonia) y de las células reproductivas (denominadas gonidias), y completa su ciclo de vida dentro de la colonia al depositar sus huevos allí (May, 1989; Nogrady, et al., 1993; Chandra \& Rawat, 2017).

Según las referencias bibliográficas revisadas, no se conoce del todo la dinámica temporal y espacial de la infección y de la presión debida al consumo del rotífero $A$. volvocicola en las colonias de Volvox; además, se desconoce dicha dinámica in situ, ya que, hasta la fecha, solo en algunos estudios se ha hecho el inventario de estas dos especies por separado a partir de muestreos de baja frecuencia e intensidad (Ganf, et al., 1983; Pennak, 1989; Shiel \& Koste, 1992; De Smet, 2006; Fontaneto \& De Smet, 2015).

El desconocimiento de la dinámica de interacción de $A$. volvocicola y Volvox se debe, probablemente, a que en los diferentes cuerpos de agua la presencia simultánea del 
rotífero y la cloroficea es esporádica, o a que los muestreos de plancton se han extendido en el tiempo (se hacen, en su mayoría, trimestralmente o bimestralmente, y solo a veces son mensuales o quincenales); además, la visualización del rotífero es posible si están presentes las colonias de Volvox (Nozaki, 2003).

Los rotíferos poseen patrones ecológicos diferentes y específicos que dependen de las condiciones del cuerpo de agua que los aloja (Pennak, 1989; Nogrady, et al., 1993, Sendacz, 1993; Lampert \& Sommer, 1997; Fernando, 2002; Estrada-Posada, 2007); sin embargo, se desconoce si en $A$. volvocicola los patrones abióticos representan algún tipo de influencia, dado que esta especie se ha considerado como un parásito o consumidor obligado (Shiel, 1995; Wallace \& Snell, 2001; Fontaneto \& De Smet, 2015), que puede depender en mayor grado de la dinámica poblacional del huésped.

En este contexto surgieron varias preguntas que constituyeron la base formal del presente estudio: ¿cuáles variables bióticas o abióticas determinan los cambios de abundancia de Volvox y del rotífero A. volvocicola en el embalse Riogrande II? ¿Cuál es la tasa de infección (presencia de rotíferos y huevos) y de consumo (daño en la membrana) del rotífero $A$. volvocicola en el alga clorofícea Volvox en el embalse Riogrande II? ¿Dichas tasas de infección y consumo cambian espacial y temporalmente?

Estas preguntas llevaron a postular la siguiente hipótesis: si el rotífero $A$. volvocicola es un parásito obligado altamente especializado y, por lo tanto, pasa la mayor parte de su ciclo de vida dentro de las colonias de Volvox, entonces su dinámica poblacional está determinada por la dinámica espacial y temporal del alga, mientras que la abundancia de Volvox en el embalse Riogrande II está controlada diferencialmente por dos fuerzas, como son las concentraciones de los nutrientes del medio abiótico (bottom-up) y la tasa de consumo del parásito (top-down), lo cual permite predecir que los florecimientos de Volvox se iniciarían en las épocas y sitios que presenten un incremento en la oferta de nutrientes, cuando y donde la tasa de infección y consumo de $A$. volvocicola sea baja, en tanto que el final del florecimiento de la clorofita se regiría tanto por la disminución de nutrientes como por el incremento de la tasa de infección y de consumo del rotífero.

\section{Materiales y métodos}

Área de estudio. El embalse Riogrande II está localizado en la parte central del departamento de Antioquia (Colombia), en la cuenca del río Grande (6 $6^{\circ} 35^{\prime} 57^{\prime \prime}-6^{\circ} 28^{\prime} 79$ " $\mathrm{N}$ y los $75^{\circ} 32^{\prime} 17^{\prime \prime}$ y $75^{\circ} 25^{\prime} 8^{\prime \prime}$ O). La cuenca aportante tiene una extensión de 1.214 hectáreas y la conforman los municipios de Donmatías, Entrerríos, San Pedro de Los Milagros y Santa Rosa de Osos. En el embalse se represan las aguas aportadas por las subcuencas del río Grande y del río Chico y la quebrada Las Ánimas (Empresas Públicas de Medellín - EPM, 2005; Mazo, et al., 2015).
El embalse hace parte del programa de aprovechamiento múltiple Riogrande II, del cual se destacan los siguientes hitos: llenado del vaso en noviembre de 1990; entrada en operación de la planta de potabilización Manantiales en 1991, e inicio de la operación de las dos centrales eléctricas (Tasajera y Niquia) en 1993 (EPM, 2005; 2019). La cota máxima del embalse está ubicada a $2.270 \mathrm{msnm}$, con un volumen total de 240,58 millones de $\mathrm{m}^{3}$, una profundidad máxima de 42,0 $\mathrm{m}$ y una media de $37,9 \mathrm{~m}$. El tiempo de residencia es de 72,8 días, la longitud del brazo más largo (río Chico) es de $10 \mathrm{~km}$. Los caudales anuales del río Chico son, en promedio, de $4,7 \mathrm{~m}^{3} / \mathrm{s}\left(1, .5-12,0 \mathrm{~m}^{3} / \mathrm{s}\right)$, y los del río Grande, de $29,0 \mathrm{~m}^{3} / \mathrm{s}\left(16,7-43,8 \mathrm{~m}^{3} / \mathrm{s}\right)$. La cuenca presenta dos periodos lluviosos, de abril a mayo y de septiembre a octubre. La precipitación anual oscila entre 1.800 y 3.000 $\mathrm{mm}$, y el rendimiento hídrico promedio es de $19 \mathrm{l} / \mathrm{s} / \mathrm{km}^{2}$ (EPM, 2005; Estrada-Posada, 2007).

Variables abióticas y bióticas. Este estudio se basó en los datos reportados por Estrada-Posada (2007) para las variables abióticas, y en las muestras de plancton recolectadas cada 10 días a lo largo de un año y almacenadas en la colección biológica del Laboratorio de Limnología del grupo LimnoBasE. Las muestras biológicas corresponden a tres sitios del embalse Riogrande II: 1) río Chico arriba (630'37" N 7532'17" O), cola del embalse, brazo del río Chico; 2) río Chico abajo (6³0’66" N 75²8’39" O), zona media, brazo del río Chico, y 3$)$ presa $\left(6^{\circ} 30^{\prime} 25^{\prime \prime} \mathrm{N}\right.$ $75^{\circ} 27^{\prime} 01^{\prime \prime} \mathrm{O}$ ), a $0,5 \mathrm{~km}$ de esta (Figura 1).

En la columna de agua en cada sitio se tomaron los perfiles verticales de temperatura y oxígeno disuelto cada $25 \mathrm{~cm}$ en los primeros diez metros $\mathrm{y}$, después, cada metro hasta el fondo. Además, se establecieron los perfiles de conductividad eléctrica, $\mathrm{pH}$, alcalinidad total y turbidez cada 5 $\mathrm{m}$ en los sitios más profundos (río Chico abajo y presa), y cada $2,5 \mathrm{~m}$ en el sitio río Chico arriba. En la zona fótica se recolectaron muestras de agua en tres profundidades diferentes (subsuperficie, mitad de la zona y límite de la zona) y se integraron para evaluar las concentraciones de sólidos totales, sólidos disueltos, sólidos suspendidos totales, sólidos volátiles y sólidos fijos, fósforo soluble, nitratos, amonios, nitrógeno total y la demanda química de oxígeno. Los métodos y las características de la masa de agua han quedado registrados en los estudios de EstradaPosada (2007), Mazo-B, et al. (2015) y Estrada-Posada \& Giraldo-Moy (2018).

Para la recolección del zooplancton se integraron cuatro profundidades (subsuperficie, $30 \%$ de la zona fótica, $60 \%$ de la zona fótica, y límite de la zona fótica) dentro de esta zona en cada sitio de muestreo, tomando un total de 20 litros con una botella de Schindler, de los cuales se extrajeron $500 \mathrm{ml}$ para evaluar la biomasa fitoplanctónica, en tanto que el volumen restante se filtró en un tamiz con poro de $60 \mu \mathrm{m}$, y la muestra se concentró en un vial de $50 \mathrm{ml}$ para determinar la composición y la densidad del zooplancton en la zona fótica (Estrada-Posada, 2007). El zooplancton 


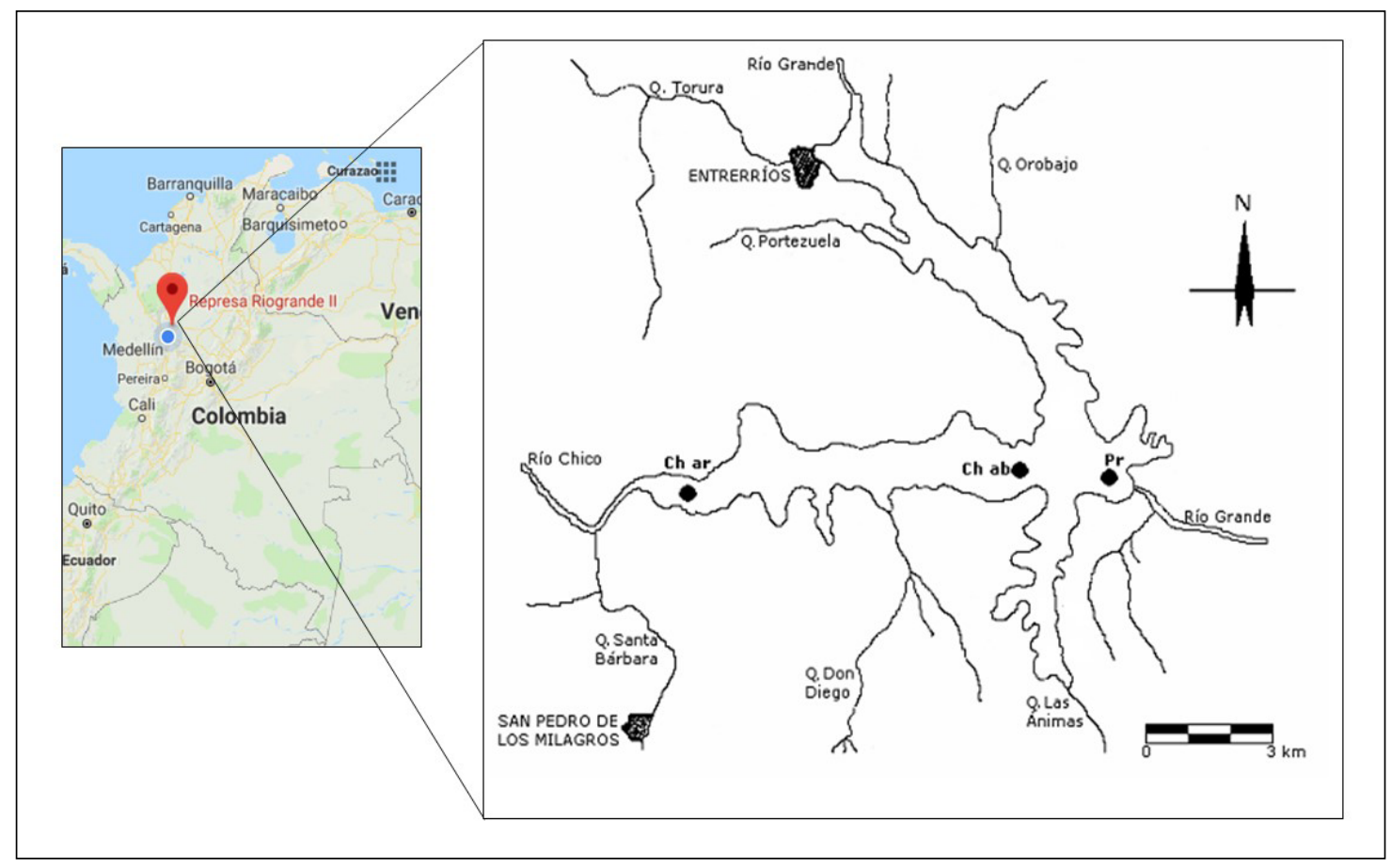

Figura 1. Sitios de muestreo en el eje del río Chico, embalse Riogrande II (Ch ar: río Chico arriba, Ch ab: río Chico abajo y Pr: presa). Fuente: Estrada-Posada \& Giraldo-Moy, 2019

se conservó en frío y, horas después, se fijó con una mezcla de azúcar y solución de Transeau (formol al $37 \%$, alcohol al $96 \%$ y agua, en proporciones de 1:3:6) para controlar las deformaciones (Haney \& Hall, 1973).

Las muestras para evaluar la biomasa fitoplanctónica se almacenaron en botellas oscuras a $4{ }^{\circ} \mathrm{C}$ para analizarlas posteriormente mediante el método de la clorofila a usando filtros de celulosa $(\varnothing=45 \mu \mathrm{m})$ y etanol caliente al $96 \%$ como solvente extractor, y acidificando el extracto con $\mathrm{HCl} 0.01$ $\mathrm{N}$ para diferenciar entre clorofila a y feopigmentos, según el protocolo de Sartory \& Grobbelaar (1984).

Para determinar la tasa de consumo o daño de la membrana de las colonias de Volvox por parte de A. volvocicola, se empleó un microscopio fotónico y una cámara de Sedgwick-Rafter, con el fin de contabilizar los mililitros necesarios para obtener 100 colonias de Volvox, ya fuesen sanas o perforadas. Simultáneamente, se registró la densidad de $A$. volvocicola y los huevos en cada colonia para estimar la tasa de infección. El número de organismos hallados y el volumen filtrado se emplearon para estimar la densidad de cada especie por litro.

El porcentaje de daño de la membrana de la colonia se estimó proyectando en hojas de papel las imágenes observadas en el microscopio para dibujar los diferentes contornos a escala de la membrana que contenía la colonia y del área dañada; toda la esfera y las áreas dañadas (huecos en la membrana) se cortaron y luego se pesaron en una balanza analítica; el área dañada se expresó como porcentaje del total del área de la esfera (Ganf, et al., 1983).
La determinación taxonómica de ambas especies se hizo usando las guías de identificación de Pennak (1989), Shiel \& Koste (1992), Nogrady, et al., 1993; Shiel (1995), Canter-Lund \& Lund (1995), y Nozaki (2003).

Análisis numérico de los datos. Se hizo un análisis estadístico descriptivo de los datos de las variables bióticas y abióticas. Para ello se usaron la media aritmética y la mediana como medidas de tendencia central, y la desviación estándar y el coeficiente de variación de Pearson como medidas de dispersión absoluta y relativa, respectivamente.

Las diferencias con significación estadística entre los sitios de muestreo y entre las fechas según las variables abióticas, se determinaron mediante un análisis de varianza (pruebas de comparaciones múltiples) y un análisis post hoc de Tukey para comparar las medias en las distribuciones que cumplieron con los supuestos exigidos por la estadística paramétrica. Las pruebas de Kruskal-Wallis y la U de MannWhitney (post hoc) se usaron para comparar las medianas en aquellas variables que después de haberlas sometido a diferentes transformaciones no cumplieron con las exigencias de los análisis paramétricos.

Las diferencias espaciales y temporales de las densidades de ambas especies y las tasas de infección y de daños de la membrana colonial, se definieron mediante un análisis de varianza (ANOVA de dos vías, espacio y tiempo) y la prueba de Newman-Keuls para la comparación múltiple de medias.

El grado de dependencia de las densidades de Volvox en relación con $A$. volvocicola y otras variables, se determinó mediante un análisis de regresión lineal simple en cada sitio, 
considerando variables como la densidad de Ascomorphella, la cantidad de huevos del rotífero, la cantidad de colonias de Volvox dañadas, y el porcentaje de daño de la membrana. Todos los análisis se efectuaron con los programas estadísticos STATGRAPHICS Plus 4.0, STATISTICA $7.0 \mathrm{y}$ MVSP 3.13.

\section{Resultados}

Con un $\mathrm{n}$ de 35 , correspondiente a las fechas de muestreo, se encontraron diferencias estadísticamente significativas para los tres sitios de muestreo en 12 de las variables abióticas evaluadas (Tabla 1). Para las formas de nitrógeno $\left(\mathrm{N}_{-} \mathrm{NO}_{3}\right.$,
$\mathrm{N}-\mathrm{NH}_{3}, \mathrm{NTK}$ ), la demanda química de oxígeno (DQO), el hierro total y el manganeso no se encontraron diferencias estadísticamente significativas. El mayor contraste se observó en los dos extremos del eje longitudinal del brazo del río Chico: el sitio río Chico arriba, ubicado en la entrada del río al embalse, con poca profundidad, y el sitio de la presa, que fue el más profundo y amplio, ubicado en el centro del embalse.

El sitio río Chico arriba presentó mayores valores de temperatura, oxígeno disuelto, conductividad eléctrica, $\mathrm{pH}$, turbidez, fósforo soluble reactivo y las diferentes formas de sólidos. En contraste, en la presa se registraron valores

Tabla 1. Tendencia central y de dispersión de las variables ambientales evaluadas en la zona fótica en tres sitios de muestreo en el embalse Riogrande II. Las primeras doce variables presentaron diferencias espaciales (pruebas ANOVA y post hoc: Tukey o U de Mann Whitney; en negrita, el promedio del sitio diferente), agosto 2002 - julio 2003

\begin{tabular}{|c|c|c|c|c|c|}
\hline \multirow{2}{*}{$\begin{array}{l}\text { Variable } \\
\text { ANOVA/post hoc }\end{array}$} & \multirow{2}{*}{$\begin{array}{l}\text { Grados de } \\
\text { libertad }\end{array}$} & \multirow[t]{2}{*}{ Estadístico } & \multicolumn{3}{|c|}{ Sitios de muestreo } \\
\hline & & & Río Chico arriba & Río Chico abajo & Presa \\
\hline \multirow{2}{*}{$\begin{array}{l}\text { Profundidad máxima (m) } \\
F=83,41, p<0,01\end{array}$} & \multirow{2}{*}{35} & Promedio & 13,13 & 26,53 & 35,52 \\
\hline & & CV (\%) & 22,41 & 24,24 & 17,00 \\
\hline \multirow{2}{*}{$\begin{array}{l}\text { Extensión de zona fótica (m) } \\
F=11,10, p<0,01\end{array}$} & \multirow{2}{*}{35} & Promedio & $\mathbf{3 , 3 7}$ & 5,53 & 5,06 \\
\hline & & CV $(\%)$ & 43,12 & 31,69 & 29,71 \\
\hline \multirow{2}{*}{$\begin{array}{l}\text { Temperatura del agua }\left({ }^{\circ} \mathrm{C}\right) \\
\mathrm{F}=6,60, \mathrm{p}<0,01\end{array}$} & \multirow{2}{*}{35} & Promedio & 22,60 & 21,30 & 20,70 \\
\hline & & CV (\%) & 4,50 & 3,20 & 4,66 \\
\hline \multirow{2}{*}{$\begin{array}{l}\text { Oxígeno disuelto (mg/l) } \\
\mathrm{X}^{2}=22,75, \mathrm{p}<0,01\end{array}$} & \multirow{2}{*}{35} & Promedio & 8,00 & 7,15 & 6,72 \\
\hline & & CV (\%) & 18,93 & 12,32 & 22,40 \\
\hline \multirow{2}{*}{$\begin{array}{l}\text { Conductividad eléctrica (uS/cm) } \\
F=16,32, p<0,01\end{array}$} & \multirow{2}{*}{35} & Promedio & 47,60 & 38,90 & 36,30 \\
\hline & & CV (\%) & 9,41 & 9,50 & 11,00 \\
\hline \multirow{2}{*}{$\begin{array}{l}\mathrm{pH} \text { (unidades) } \\
\mathrm{X}^{2}=49,63, \mathrm{p}<0,01\end{array}$} & \multirow{2}{*}{35} & Promedio & 9,61 & 9,11 & 8,92 \\
\hline & & CV (\%) & 3,70 & 6,44 & 6,56 \\
\hline \multirow{2}{*}{$\begin{array}{l}\text { Turbidez (FTU) } \\
\mathrm{X}^{2}=19,49, \mathrm{p}<0,01\end{array}$} & \multirow{2}{*}{35} & Promedio & 28,55 & 9,31 & 10,55 \\
\hline & & CV (\%) & 257,30 & 57,48 & 66,51 \\
\hline \multirow{2}{*}{$\begin{array}{l}\text { Sólidos totales }(\mathrm{mg} / \mathrm{L}) \\
\mathrm{X}^{2}=19,04, \mathrm{p}<0,01\end{array}$} & \multirow{2}{*}{35} & Promedio & 80,60 & 68,60 & 64,80 \\
\hline & & CV (\%) & 24,40 & 20,26 & 20,79 \\
\hline \multirow{2}{*}{$\begin{array}{l}\text { Sólidos suspendidos total }(\mathrm{mg} / \mathrm{L} \text { ) } \\
\mathrm{X}^{2}=27,06, \mathrm{p}<0,01\end{array}$} & \multirow{2}{*}{35} & Promedio & 16,23 & 7,36 & 7,47 \\
\hline & & CV (\%) & 102,63 & 31,76 & 33,92 \\
\hline \multirow{2}{*}{$\begin{array}{l}\text { Sólidos volátiles totales }(\mathrm{mg} / \mathrm{L}) \\
\mathrm{F}=7,17, \mathrm{P}<0,01\end{array}$} & \multirow{2}{*}{35} & Promedio & 53,20 & 43,40 & 37,80 \\
\hline & & CV (\%) & 27,45 & 30,14 & 30,57 \\
\hline \multirow{2}{*}{$\begin{array}{l}\text { Sólidos disueltos }(\mathrm{mg} / \mathrm{L}) \\
\mathrm{X}^{2}=10,19, \mathrm{p}<0,05\end{array}$} & \multirow{2}{*}{35} & Promedio & 64,33 & 61,25 & 57,32 \\
\hline & & CV (\%) & 22,44 & 22,47 & 22,14 \\
\hline \multirow{2}{*}{$\begin{array}{l}\text { Fósforo soluble reactivo }(\mathrm{mg} / \mathrm{L}) \\
\mathrm{F}=6,24, \mathrm{p}<0,01\end{array}$} & \multirow{2}{*}{11} & Promedio & 0,14 & 0,07 & 0,04 \\
\hline & & CV (\%) & 54,70 & 129,88 & 218,53 \\
\hline \multirow{2}{*}{$\begin{array}{l}\text { Nitratos }(\mathrm{mg} / \mathrm{L}) \\
\mathrm{p}>0,05 \text { D.N.S. }\end{array}$} & \multirow{2}{*}{11} & Promedio & 1,57 & 1,56 & 1,52 \\
\hline & & CV (\%) & 61,48 & 62,25 & 61,23 \\
\hline \multirow{2}{*}{$\begin{array}{l}\mathrm{NH}_{4}(\mathrm{mg} / \mathrm{L}) \\
\mathrm{p}>0,05 \text { D.N.S. }\end{array}$} & 11 & Promedio & 0,38 & 0,19 & 0,26 \\
\hline & 11 & CV (\%) & 104,22 & 144,88 & 106,11 \\
\hline
\end{tabular}

D.N.S.: diferencias estadísticamente no significativas, en negrilla los valores que mostraron diferencias significativas 
menores de las variables antes mencionadas y mayor profundidad. El sitio río Chico abajo presentó valores medios o menores para la mayoría de las variables, y los mayores promedios para la extensión de la zona fótica (Tabla 1). Los tres sitios de muestreo presentaron estratificación térmica y química en el eje gravedad-luz, con mezcla del agua metalimnética dentro de la capa superficial y con hipolimnio anóxico durante el año de muestreo (Mazo, et al., 2015).

Las concentraciones promedio de clorofila a presentaron diferencias estadísticamente significativas (ANOVA, $\mathrm{F}=22,30 ; \mathrm{p}<0,01, \mathrm{gl}=35)$. La prueba $\mathrm{U}$ de Mann-Whitney permitió determinar que el sitio río Chico arriba fue diferente y presentó los mayores valores (109,3 ug/l) en los meses de abril y mayo de 2003, llegando a ser seis veces más que el promedio registrado para la presa $(18,5 \mathrm{ug} / \mathrm{l})$ y cuatro veces mayor que río Chico abajo $(25,9 \mathrm{ug} / \mathrm{l})$.

Las densidades de las colonias de Volvox fueron mayores en el sitio río Chico arriba, con picos de hasta $2.547 \mathrm{ind} / 1$, tanto que en los sitios río Chico abajo y presa las densidades máximas fueron de 342 y $125 \mathrm{ind} / 1$, respectivamente. Estos picos de densidades de Volvox y otros menores se registraron a finales de octubre de 2002 (río Chico abajo), noviembre de 2002 (en los tres sitios), primera quincena de abril y junio de 2003 (río Chico arriba y presa) (Figura 2). Además, Volvox se detectó en 32 de los 35 muestreos realizados en, por lo menos, un sitio de muestreo. La mayor frecuencia de colonias de Volvox se observó en río Chico arriba, con 26 fechas de muestreo, río Chico abajo, con 19, y presa, con 17.

Con base en estos hallazgos, la densidad de Volvox presentó diferencias estadísticamente significativas en la dimensión espacial (ANOVA, $\mathrm{F}=4,43 ; \mathrm{p}<0,01$ ). En este sentido, la prueba de Newman-Keuls indicó que río Chico arriba fue diferente (por presentar las mayores densidades), en tanto que los sitios río Chico abajo y presa no mostraron diferencias significativas entre ellos y registraron las menores densidades (Figura 2).

También se encontraron diferencias estadísticamente significativas en la dimensión temporal para las densidades de Volvox (ANOVA, F=2,59; $\mathrm{p}<0,01$ ). La prueba de Newman-Keuls indicó que solo noviembre de 2002, cuando se presentó el mayor pico de densidad en el sitio río Chico arriba, difirió en cuanto a las densidades de Volvox.

Ascomorphella volvocicola se encontró en los tres sitios de muestreo en las diferentes épocas del año, coincidiendo con la presencia de Volvox. En los análisis microscópicos no se observaron individuos de vida libre del rotífero, todos estaban dentro de las colonias de Volvox. La densidad de A. volvocicola presentó un máximo de 166,67 ind/l en río Chico arriba (noviembre de 2002), de 12,75 ind/1 en presa (junio de 2003) y de 11,25 ind/1 en río Chico abajo (octubre de 2002) (Figura 2).

Se observaron diferencias temporales y espaciales de la abundancia de $A$. volvocicola, con picos de densidad en las mismas fechas que Volvox: a finales de octubre de 2002 (en río Chico abajo), noviembre de 2002 (en los tres sitios), y primera quincena de abril de 2003 y de junio de 2003 (en río Chico arriba y presa) (Figura 2). Durante 16 fechas de muestreo no se registraron individuos de $A$. volvocicola en ninguno de los tres sitios.

Los huevos de $A$. volvocicola, al igual que las colonias de Volvox y el rotífero, presentaron tres picos de densidad en los mismos meses, con mayor variabilidad en los datos de densidad en el sitio río Chico arriba (comparado con los sitios río Chico abajo y presa) (Figuras 3 y 4 ). La cantidad de huevos por colonia varió entre cero (0) y cuatro (4).

En 13 fechas de muestreo $(37,14 \%)$ las colonias de Volvox no presentaron infestación de A. ascomorphela; es decir, no se observaron evidencias de consumo o daños en la membrana, ni presencia del rotífero o sus huevos (Figuras 2 y 3 ).

En cuanto al daño de la membrana de Volvox, se registraron diferentes porcentajes de consumo (entre 5 y $52 \%$ ) (Figura 3), pero sin diferencias estadísticamente significativas entre los sitios de muestreo; sin embargo, se encontraron diferencias en el tiempo (ANOVA, $F=5,03 ; p<0,01$ ), siendo noviembre de 2002 el mes diferente (según la prueba de Newman-Keuls post hoc), pues se observó mayor porcentaje de daño en la membrana.

Durante los picos de densidad de Volvox, todas las colonias presentaron algún tipo de daño en la membrana o infestación (entendida como presencia de huevos o adultos de A. volvocicola) en el sitio de la presa, mientras que en río Chico arriba se observó que el $62,60 \%$ de las colonias presentó daños o infestación, y en río Chico abajo se registró el menor porcentaje de daño o infestación $(10,50 \%)$.

En cuanto a las tasas de incremento o descenso de las abundancias del rotífero y el alga, se encontró que $A$. volvocicola apareció luego de Volvox y ambas disminuyeron al mismo tiempo, patrón que se cumplió para todos los sitios de muestreo (Figura 2).

Mediante el análisis de regresión lineal simple se estableció que no hubo relaciones significativas de las densidades de Volvox ni de A. volvocicola con factores abióticos, en tanto que sí se encontraron relaciones significativas de Volvox con algunas variables bióticas. Estos análisis evidenciaron la relación de las densidades de Volvox con la variación de la abundancia del rotífero (en los tres sitios), con el número de huevos de $A$. volvocicola (en río Chico arriba y Chico abajo), el porcentaje de daño de la membrana (en río Chico abajo) y la cantidad de individuos de Volvox dañados (en río Chico arriba y presa) (Tabla 2).

\section{Discusión}

El embalse Riogrande II es un cuerpo de agua eutrófico con las mayores concentraciones de nutrientes (fósforo), clorofila a y sólidos en la cola del embalse (río Chico arriba), y con menor concentración de estas variables hacia los tramos más profundos y leníticos (río Chico abajo y presa). Este patrón está relacionado con la retención, la sedimentación y la mineralización del material aportado por la cuenca 


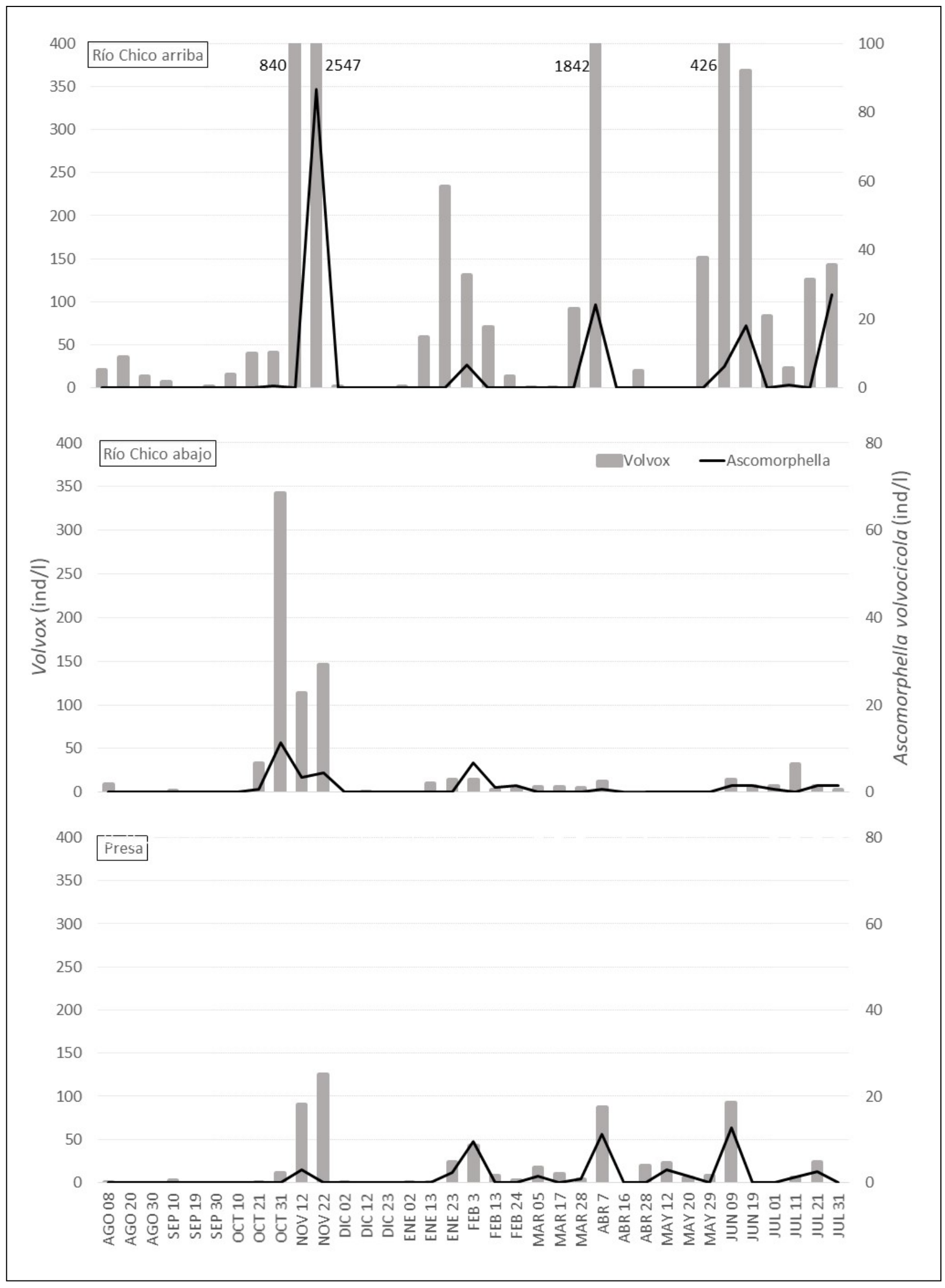

Figura 2. Dinámica temporal y espacial de la densidad de colonias de Volvox y de individuos de Ascomorphella volvocicola en el embalse Riogrande II, agosto 2002 - julio 2003. En tres sitios de monitoreo: río Chico arriba, río Chico abajo y presa 


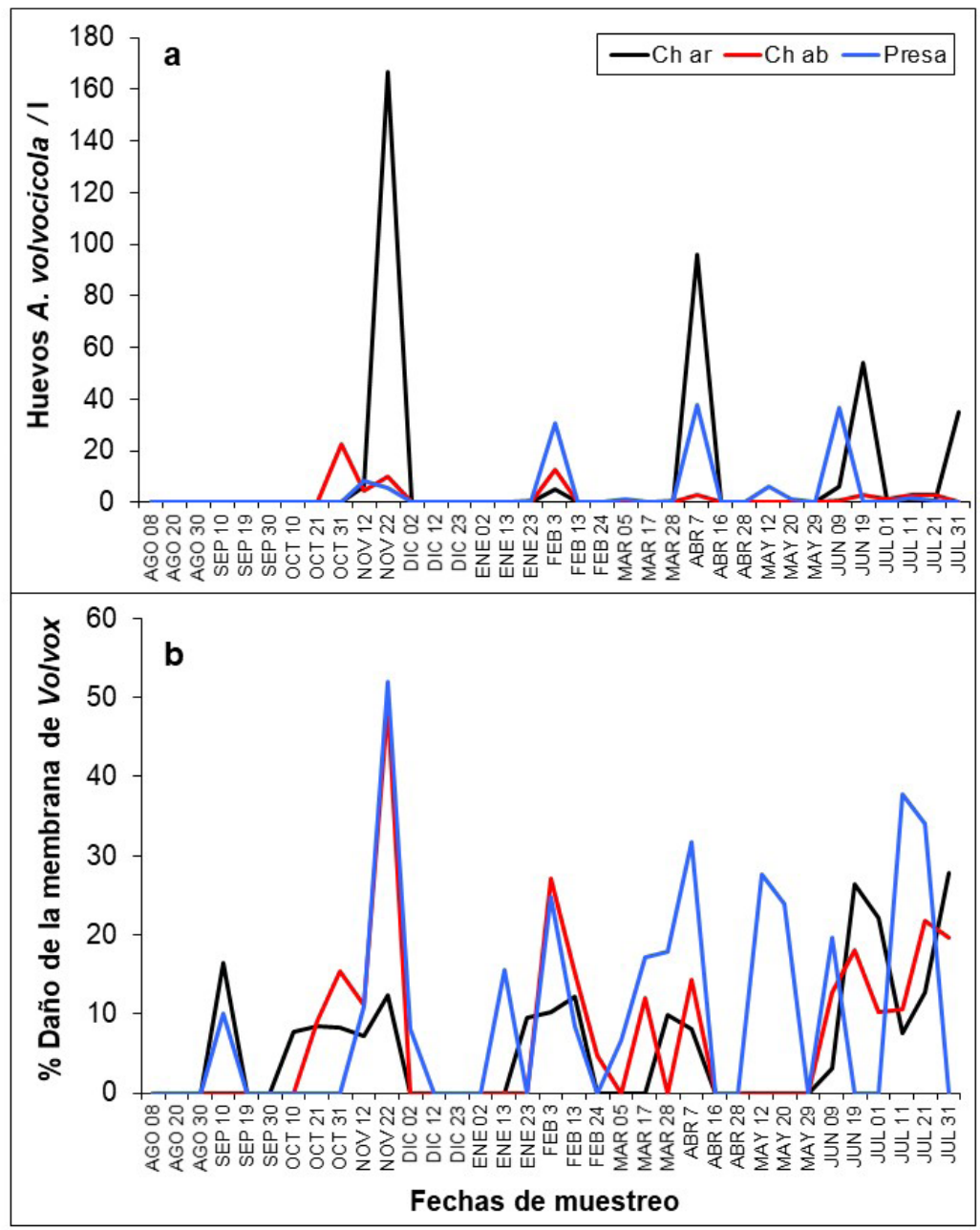

Figura 3. Registros de infestación por Ascomorphella volvocicola en colonias de Volvox en tres sitios de muestreo en el embalse Riogrande II. a. Densidad de huevos del rotífero. b. Porcentaje de daño en la membrana, agosto 2002 - julio 2003. Ch ar: río Chico arriba, Ch ab: río Chico abajo y Pr: presa

afluente, que se presenta en el primer tramo del recorrido de los ríos represados (Armengol, et al., 1999, Espíndola, et al., 2000, Duggan, et al., 2002, Armengol, et al., 2009). En el presente estudio, dicho patrón condujo a predecir inicialmente que las poblaciones de Volvox del embalse Riogrande II presentarían densidades diferenciadas en el espacio gracias a la heterogeneidad en las concentraciones de los nutrientes.

Las tendencias físicas y químicas encontradas en el brazo del río Chico fueron heterogéneas en el eje longitudinal. Estas condiciones abióticas favorecen el desarrollo diferente tanto en el espacio como en el tiempo del fitoplancton y del zooplancton (Lampert \& Sommer, 1997), lo que explicaría la mayor biomasa de fitoplancton (cuantificada como clorofila a) en el sitio río Chico arriba. Sin embargo, según los resultados de los análisis estadísticos, las diferencias en el ambiente, caracterizado a partir de 20 variables, no parecen relacionarse con la dinámica de las poblaciones de Volvox y A. volvocicola.
Algunos reportes señalan que las especies de Volvox se ven afectadas por la concentración de calcio en el agua (variable no evaluada en esta investigación), y pueden ser estimuladas a desplazarse en la columna de agua (eje vertical) cuando el ambiente es eutrófico y presentan orientación fototáctica (Moss, 1972; Ganf, et al., 1983, Reynolds, 2006).

Investigadores como Lampert \& Sommer (1997) y Drescher, et al. (2009) han reportado la habilidad de las colonias de Volvox para migrar verticalmente, llegando a desplazarse a una velocidad de $1 \mathrm{~mm} \mathrm{~s}^{-1}$ (Sommer \& Gliwicz, 1986). Cualquier factor de presión o de insuficiencia, como el de los nutrientes o la luz, puede ser resuelto por las colonias de Volvox, ya que se ha reportado que durante el día estas algas permanecen en la zona fótica para captar la luz necesaria y en la noche migran a las profundidades donde se encuentran disponibles los nutrientes (Sommer \& Gliwics, 1986).

Los resultados obtenidos indican que $A$. volvocicola es un endoparásito obligado que predomina en los ambientes y 


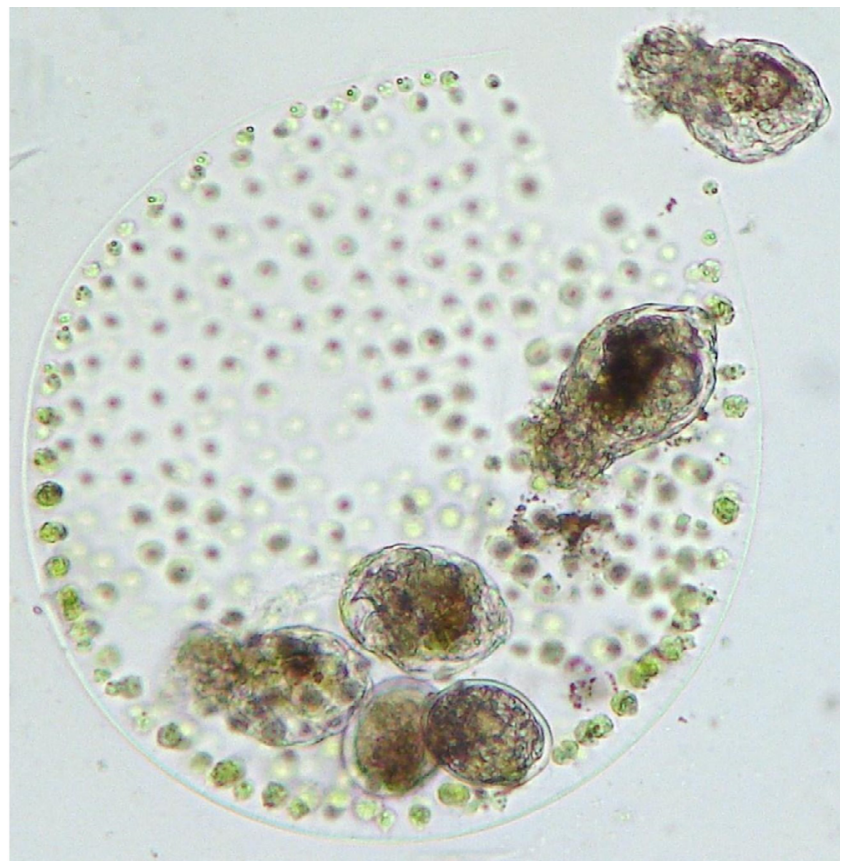

Figura 4. Ascomorphella volvocicola parasitando Volvox. Fotografía: 400X, tomada por las autoras, 2007

Tabla 2. Valores obtenidos en las regresiones lineales para describir las relaciones entre la densidad de Volvox y las variables bióticas en cada sitio $(\mathrm{p}<0,01)$

\begin{tabular}{llcc}
\hline Sitio & Variable & r & $\begin{array}{c}\text { Tipo de } \\
\text { relación }\end{array}$ \\
\hline \multirow{2}{*}{$\begin{array}{l}\text { Río Chico } \\
\text { arriba }\end{array}$} & Densidad Ascomorphella & 0,86 & Lineal \\
\cline { 2 - 4 } & Huevos & 0,93 & Lineal \\
\hline \multirow{2}{*}{$\begin{array}{l}\text { Río Chico } \\
\text { abajo }\end{array}$} & Densidad Ascomorphella & 0,86 & Lineal \\
& Huevos & 0,87 & Lineal \\
\hline \multirow{2}{*}{ Presa } & Porcentaje de daño membrana & 0,59 & $\sqrt{\mathrm{Y}}$ \\
& Densidad Ascomorphella & 0,68 & $\sqrt{\mathrm{Y}}$ \\
\hline
\end{tabular}

en las épocas en que aparecen las colonias de Volvox, ya que no se encontró fuera de las colonias ni en épocas diferentes. La aparición de este rotífero ocurre después de que esta clorofícea aflora y la densidad disminuye cuando Volvox comienza a decaer. Los escasos reportes de A. volvocicola en los inventarios de este estudio pueden deberse a que no apareció en todas las ocasiones en que floreció Volvox. En un tercio de los muestreos en el embalse Riogrande II no se observaron estos rotíferos, sin embargo, se presentaban colonias de Volvox. Los picos de densidad de A. volvocicola posiblemente están asociados con mayores densidades de su hospedero, de ahí su ausencia cuando las densidades de Volvox eran bajas.
La presencia de colonias de Volvox que portaban únicamente huevos puede indicar que el rotífero tiene un patrón de uso itinerante de las colonias de Volvox, que responde a la ruptura de la membrana, el consumo de una parte de esta, la reproducción y la postura de los huevos en el interior de la colonia y, luego, el abandono del alga para repetir el proceso en otras colonias y así continuar su ciclo de vida, hecho también reportado por Ganf, et al. (1983). Este tipo de ciclo de vida ambulante de $A$. volvocicola en el embalse Riogrande II puede llegar a producir el daño de hasta el $60 \%$ de la membrana en las colonias de Volvox parasitadas, posiblemente como resultado de varios individuos parásitos. Esta estrategia ambulante también permite que quede suficiente alimento para los rotíferos hijos cuando salen de sus huevos.

En cuanto al rotífero $A$. volvocicola, se concluyó que su presencia, densidad y reproducción no dependieron de las condiciones abióticas del embalse, sino de la presencia de Volvox, condición propia de los parásitos (Ebert, 2005), los depredadores o los herbívoros altamente especializados (Lampert \& Sommer, 1997). Puesto que, en ningún momento, el rotífero parásito $A$. volvocicola redujo la abundancia de colonias de Volvox, se infiere que tanto el alga clorofita como el rotífero tenían suficientes recursos. El avance de la infección o su eficiencia dependen de la diferencia entre la tasa de incremento de las poblaciones del hospedero y del parásito; si el hospedero presenta limitaciones ambientales, su tasa de incremento tiende a disminuir, lo que haría vulnerable la estabilidad de la población ante la infección del parásito (Lampert \& Sommer, 1997).

La estrecha relación entre $A$. volvocicola y Volvox lleva a postular que la presencia de este rotífero estuvo completamente ligada a la aparición y florecimento de Volvox, como también se ha reportado en cuerpos de agua de otras latitudes (Ganf, et al., 1983). En las diferentes especies de fitoplancton existentes en el embalse Riogrande II no se encontraron individuos de $A$. volvocicola infectando otras algas, por lo tanto, esta especie de rotífero es altamente especializada y solo infecta al género Volvox.

Autores como Canter-Lund \& Lund (1995) consideran a Volvox como el hábitat de $A$. volvocicola. Wallace \& Snell (2001) hacen referencia a este rotífero como una especie depredadora dentro de la colonia de Volvox (Tabla 3). Para otros autores, como Ganf, et al. (1983), este rotífero es un parásito cuya presencia se relaciona estrechamente con la de Volvox, pues al igual que en el presente estudio, no hallaron individuos de vida libre ni asociados con otras algas. Según los resultados del presente estudio, la especie A. volvocicola se comporta como un parásito de alta especificidad y dependencia que aparece solo durante los florecimientos de Volvox y, además, tiene la capacidad de ocasionar daños de magnitud importante sobre la membrana.

Teniendo en cuenta los resultados, y retomando la hipótesis formulada, esta debe rechazarse en cuanto al control de los nutrientes y del endoparásito sobre Volvox, 
Tabla 3. Consideración del uso dado por A. volvocicola a las colonias de Volvox según diferentes autores

\begin{tabular}{|c|c|c|c|c|}
\hline Autor & Año & Hábitat & Hospedero-parásito & Presa-depredador \\
\hline Ehrenberg (May, 1989) & 1835 & & $\mathbf{x}$ & \\
\hline Gosse & 1852 & & $\mathbf{x}$ & \\
\hline Williams & 1852 & & $\mathbf{x}$ & \\
\hline Edmonson & 1959 & $\mathbf{x}$ & & \\
\hline Pennak & 1989 & & $\mathbf{x}$ & \\
\hline Ganf, et al. & 1983 & & $\mathbf{x}$ & \\
\hline Shiel \& Koste & 1992 & & $\mathbf{x}$ & \\
\hline Canter-Lund \& Lund & 1995 & $\mathbf{X}$ & & \\
\hline Wallace \& Snell & 2001 & & & $\mathbf{x}$ \\
\hline Fontaneto \& Smet & 2015 & & $\mathbf{x}$ & \\
\hline
\end{tabular}

y aceptarse en lo que respecta al hecho de que la dinámica poblacional del rotífero $A$. volvocicola está determinada por la dinámica del alga, lo cual respondería al hecho de que Volvox puede escoger su entorno, mientras que $A$. volvocicola, al ser un endoparásito altamente especializado, no puede hacerlo, pues cumple su ciclo de vida dentro de las colonias de Volvox. La habilidad de migración de Volvox sería una de las razones por las que el rotífero no diezma las poblaciones de esta alga; otra podría ser la alta disponibilidad de nutrientes en el sitio río Chico arriba, lo que ayuda a que dicha alga mantenga una tasa de reproducción mayor que la del rotífero parásito.

Por último, en el embalse Riogrande II no existe una regulación de las densidades de Volvox según los nutrientes, ya que este se encuentra en una condición eutrófica, lo que permite que haya disponibilidad todo el tiempo, con predominio de esta condición en la cola del río Chico (EstradaPosada, 2007; Zabala, 2013 y Villabona, et al., 2015).

\section{Agradecimientos}

Las autoras agradecen a los funcionarios del embalse Riogrande II y de las Empresas Públicas de Medellín que hicieron posible las jornadas de toma de muestras. Al profesor John Jairo Ramírez Restrepo, por facilitar el espacio y los equipos necesarios para el procesamiento de las muestras. Al profesor Abel Díaz por el apoyo en el análisis estadístico. Al equipo humano y académico del Laboratorio de Limnología del grupo LimnoBasE, del Instituto de Biología de la Universidad de Antioquia, en cuya colección biológica se mantuvieron las muestras de plancton recolectadas por Estrada-Posada (2007). A los revisores anónimos por sus oportunos aportes.

\section{Contribución de las autoras}

Las autoras declaran haber participado activamente en cada una de las fases del estudio y la preparación del manuscrito y en todo lo concerniente a la gestión, procesamiento, análisis y discusión de la información aquí descrita.

\section{Conflicto de intereses}

Las autoras declaran no tener conflictos de intereses que puedan de alguna manera influir en la transparencia u objetividad del proceso de revisión. Tampoco tienen conflictos de intereses personales ni de tipo financiero.

\section{Referencias}

Armengol, J., García, J.C., Comerma, M., Romero, M., Dolz, J., Roura, M. Han, B. P., Vidal, A., Simek, K. (1999). Longitudinal Processes in Canyon Type Reservoirs: The case of Sau (N.E. Spain). En: Theoretical Reservoir Ecology and its Aplicactions. Tundisi J. G. y Straskraba M. (eds.). p. 313-345.

Armengol, J., Rodríguez, J. J., García, J.C., Ordónez, J., Marcé, R. (2009). La gestión de los embalses en relación a la calidad del agua en condiciones de sequía extrema. Ingeniería del Agua. 16 (4): 285-294.

Brumley, D. R., Polin, M., Pedley, T. J., \& Goldstein, R. E. (2015). Metachronal waves in the flagellar beating of Volvox and their hydrodynamic origin. Journal of the Royal Society Interface. 12 (108): 20141358.

Canter-Lund, H. M., Lund, J. W.G. (1995). Freshwater algae: Their microscopic world explored. Bristol: Biopress. p. 380.

Chandra, S., Rawat, D. (2017). Species richness estimate of freshwater rotifers (Animalia: Rotifera) of western Maharashtra, India with comments on their distribution. Current Science. 112 (4): 695-698.

De Smet, W. H. (2006). Asciaporrectidae, a new family of Rotifera (Monogononta: Ploima) with description of Asciaporrecta arcellicola gen. et sp. nov. and A. difflugicola gen. et sp. nov. inhabiting shells of testate amoebae (Protozoa). Zootaxa. 1339: 31-49.

Drescher, K., Leptos, K. C., Tuval, I., Ishikawa, T., Pedley, T. J., Goldstein, R. E. (2009). Dancing Volvox: Hydrodynamic bound states of swimming algae. Physical Review Letters. 102 (16): 168101.

Duggan, I. C., Green, J. D., Shiel, R. J. (2002). Distribution of rotifer assemblages in North Island, New Zeland, lakes: Relationships to environmental and historical factors. Freshwater Biology. 47: 195- 206. 
Ebert, D. (2005). Ecology, Epidemiology, and Evolution of Parasitism in Daphnia. National Library of Medicine (US), National Center for Biotechnology Information. p. 98.

Edmondson, W. T. (1959). Freshwater Biology. New York, E.U.A. John Willey \& Sons, Inc. p. 1248.

EPM - Empresas Públicas de Medellín. (2005). Cincuenta años de hidrometeorología en Empresas Públicas de Medellín. Revista Hidrometeorológica. 1 (1): 1-150.

EPM - Empresas Públicas de Medellín. (2019). Institucional, nuestras plantas. Fecha de consulta: 20 de julio de 2019. Disponible en https://www.epm.com.co

Espíndola, E.L.G., Matsumura-Tundisi, T., Rietzler, A. C., Tundisi, J. G. (2000). Spatial heterogeneity of the Tucurui reservoir (State of Parana, Amazonia, Brazil) and the distribution of zooplanktonic species. Revista Brasilera de Biología. 60: 179-194.

Esteves, F. A. (2011). Fundamentos de limnología. 3a. Edición. Río de Janeiro. Brazil. Editora Interciencia Ltda. p. 790.

Estrada-Posada A. L. (1999). Variação espacial e temporal da comunidade zooplanctônica do Reservatório "La Fé", Antioquia, Colombia. Tesis de maestría. [Sao Paulo (Brasil)]: Universidad de São Paulo. p. 78.

Estrada-Posada, A. L. (2007). Variación de la estructura y la distribución del zooplancton en función de gradientes longitudinales abióticos y bióticos en el embalse Riogrande II (Antioquia), Colombia. Tesis de Doctorado en Biología, Instituto de Biología, Universidad de Antioquia.

Estrada-Posada, A. L. \& Giraldo-Moy, M. A. (2018). Dinámica de la dieta de Asplanchna girodi (De Guerne. 1888) en un embalse tropical, Colombia. Orinoquia - Universidad de los Llanos - Villavicencio, Meta. Colombia. 22 (2): 189-203.

Fernando, C.H. (2002). A guide to tropical freshwater zooplankton: Identification, ecology and impact on fisheries. Leiden, The Netherlands. Backhuys Publishers.

Fontaneto, D. \& De Smet, W. (2015). 4. Rotifera. Handbook of zoology, Gastrotricha and Gnathifera. 217-300.

Ganf, J. G. G., Shiel, R. J., Merrick, C. J. (1983). Parasitism: The Possible Cause of the Collapse of a Volvox Population in Mount Bold Reservoir, South Australia. Aust. Journal Marine. Freshwater. Res. 34: 489-94.

Gosse, P.H. (1852). On the Notommata parasita Ehrbg., a rotiferous animal inhabiting the spheres of Volvox globator. Trans. Microsc. Soc. London. 3: 143-146.

Haney, J. F. \& Hall, D. J. (1973). Sugar-coated Daphnia: A preservation technique for Cladocera. Limnology Oceanography. 18: 331-333.

Kirk, D. L. (2004). Volvox. Current biology. 14 (15): R599-R600.

Kirk, D. L. (2005). Volvox: A search for the molecular and genetic origins of multicellularity and cellular differentiation. Vol. 33. Cambridge University Press. p. 381.

Koste, W. (1978). ROTATORIA. Uberordnung Monogononta. Vol. II. Berlín, Alemania. p. 234.

Lampert, W. \& Sommer, U. (1997). Limnoecology: The ecology of lakes and streams. New York, Oxford University Press. p. 382 .

Margalef, R. (1983). Limnología. Ediciones Omega, S.A. Barcelona. p. 1010.

Martínez-Silva, P. (2015). Variación espacio-temporal de microalgas acuáticas del embalse de Betania-Huila y su relación con la calidad del agua. Revista Intropica. 10: 11-19.
Martínez Silva, P., Muñoz Y. J. L., Losada B. L. C., Rodríguez, S. R. (2018). Composition of the Phytoplankton Community in the First Stages of the Quimbo Dam Life Cycle in Huila-Colombia. Contemporary Engineering Sciences. 11 (19): 907-924.

May, L. (1989). Epizoic and parasitc rotifers. Hydrobiologia. 186/187: 59-67.

Mazo, B. D., Ramírez-Restrepo, J. J., Díaz-C, A. (2015). Caracterización física y química del embalse Riogrande II (Antioquia), Colombia. Actualidades Biológicas, 37 (103): $155-168$

Moss, B. (1972). The Influence of Environmental Factors on the Distribution of Freshwater Algae: An Experimental Study: I. Introduction and the Influence of Calcium Concentration. The Journal of Ecology. 60 (3): 917-932.

Nogrady, T., Wallace, R. L., Snell, T. W. (1993). Rotifera: Biology, ecology and systematics. Guides to the identification of the Microinvertebrates of the continental waters of the World. The Netherlands. SPB Academic Publishing. p. 142.

Nozaki, H. (2003). Flagellated green algae. En: Wehr, J.D., Sheath. R.G. (Eds). Freshwater Algae of North America: Ecology and Classification. Academic Press. p. 918.

Pennak, R. W. (1989). Freshwater invertebrates of the United States. Tercera edición. New York, E.U.A. John Willey y Sons, Inc. p. 628.

Ramírez, J. J. (1986). Fitoplancton de red en el embalse de El Peñol, Colombia. Actualidades biológicas. 15 (56): 2-13.

Reynolds, C. S. (2006). The ecology of Phytoplankton. Cambridge University Press. p. 535.

Ricklefs, R. E. (1998). Invitación a la ecología: la economía de la naturaleza. España. Ed. Médica Panamericana S.A. p. 692.

Sánchez, C. (2000). Origen y evolución del parasitismo. Academia de Ciencias Exactas, Físicas, Químicas y Naturales de Zaragoza. Sdad. Coop. De Artes.

Sartory, D. P. \& Grobbelaar, J. U. (1984). Extraction of chlorophyll a from freshwater phytoplankton for spectrophotometric analysis. Hydrobiologia. 114: 177-187.

Sendacz, S. (1993). Distribuição geográfica de alguns organismos zooplanctônicos na America do Sul. Acta Limnologica Brasiliensia. 6: 31-41

Shiel, R.J. \& Koste, W. (1992). Rotifera from Australian Inland waters Vlll. Trichocercidae (Monogononta). Transactions of the Royal Society of South Autralia. 116 (1):1-27.

Shiel, R. J. (1995). A guide to identification of rotifers, cladocerans and copepods from Australian inland waters. Albury, Australia. Co-operative Research Centre for Freshwater Ecology. p. 144.

Sommer, U.\& Gliwicz, Z. M. (1986). Long range vertical migration of Volvox in tropical Lake Cahora Bassa (Mozambique). Limnology and Oceanography. 31 (3): 650-653.

Stiling, P.D. (1996). Ecology: theories and Applications. Tercera edición. University of South Florida. Prentice Hall, New Jersey. p. 539.

van Donk, E. \& Voogd, H. (1998). Control of Volvox blooms by Hertwigia, a rotifer. Internationale Vereinigung für theoretische und angewandte Limnologie: Verhandlungen. 26 (4): 1781-1784

Villabona-González, S. L., Buitrago-Amariles, R. F., RamírezRestrepo, J. J., Palacio-Baena, J. A. (2014). Biomasa de rotíferos de dos embalses con diferentes estados tróficos 
(Antioquia, Colombia) y su relación con algunas variables limnológicas. Actualidades Biológicas. 36 (101): 149-162.

Villabona-González, S. L., Ramírez-Restrepo, J. J., PalacioBaena, J. A., Bonecker, C. C. (2015). Respuesta de la biomasa zooplanctónica a los gradientes de estado trófico y precipitación de un embalse tropical. Revista de la Academia Colombiana de Ciencias Exactas, Físicas y Naturales. 39 (152): 374-388.

Wallace, R. L. \& Snell, T. W. (2001). Phylum Rotifera. En: Thorp, J. H., Covich, A. P. Ecology and classification invertebrates of North American Freshwater Invertebrates. USA. 3a ed. Academic Press. 173-236.

Wetzel, R. G. (2001). Limnology: Lake and River Ecosystems. USA. 3 a ed. Academic Press. p. 1006.

Williams, J. (1852). On the ocurrence of parasitic Rotifera in Volvox globator. Trans. Microsc. Soc. London. 3: 129-131.

Zabala, A.M. (2013). Evaluación del estado trófico del embalse Riogrande II Antioquia, Colombia) a través del comportamiento espacial y temporal de los nutrientes (Trabajo de grado). Universidad de Antioquia. Medellín. 